\title{
Are Mathematical Relationships Represented by Geometries in Some Functional State Space?
}

\author{
Andy E. Williams, Nobeah Foundation, Nairobi, Kenya
}

\begin{abstract}
:
Human-Centric Functional Modeling is considered in this paper to be a branch of network theory which hypothesizes that all systems can be represented as having a set of functions through which the system might transition from one functional state to another. These states are referred to as "functional states" because they are described solely in terms of the functions available to transition to adjacent states, rather than being described in terms of any entities that might implement those functions. All possible behaviors of the system are then hypothesized to be described by a graph containing a network of nodes representing such functional states, where those nodes are connected by edges representing the processes through which those functional states might transition between each other. Modeling human cognition as moving through a "space of concepts" or "conceptual space" that forms the functional state space of the cognitive system and that has the capacity to represent all possible concepts and reasoning, it has been hypothesized that all information and therefore all mathematical proofs and other logic can be represented in this space. Modeling human conscious self-awareness as moving through an "awareness space" or "space of awareness's" that represents the functional state space of the consciousness, it has been hypothesized that awareness of any mathematical or other truth, whether or not yet identified or proven, can be represented as a potential awareness in that space. This paper outlines the hypotheses that in the limit of infinite ability to navigate logic through understanding or reasoning processes (infinite intelligence) mathematical logic is represented by a geometry in conceptual space, and that in the limit of infinite ability to be aware of truth (infinite awareness), mathematical truth is represented as a geometry in awareness space. This paper also outlines some of the questions that must be answered in order to validate these hypotheses.
\end{abstract}

\section{Introduction}

Outside of mathematics, network theory [1] is the study of graphs as a representation of either symmetric relations or asymmetric relations between discrete objects. Within mathematics, network theory is part of graph theory [2]. Human-Centric Functional Modeling [3] is a network theory that represents all systems as moving through a "functional state space". Defining each system as having a set of functions, each system then can potentially transition from one state defined in terms of these functions (that is, can transition from one "functional state") to another. All possible behaviors of the system can then potentially be represented by a graph containing a network of nodes representing all functional states, connected by edges consisting of the processes by which one functional state can potentially transition to any adjacent state. These spaces may be open in that new functional states can be added or existing functional states removed, and in that new processes to transition to those new states can be added, and processes to transition to functional states can also be removed. These processes are represented as potentially consisting of some combination of some basic set of functions that "spans" all possible processes in that functional state space in that this basic set can be used to express any such process. Human-Centric Functional Modeling has been used to define a hypothetical model for the complete set of behaviors that the human cognitive system can possibly have, that is, a model of all possible concepts and reasoning that human cognition can possibly contemplate. This hypothetical space of concepts or "conceptual space" is an open graph containing a network of all possible concepts in which each concept is connected other concepts by available reasoning processes. Assuming this graph can contain all possible concepts and reasoning, it is a complete representation of the human meaning of all information, that is, a complete semantic model. Similarly, Human-Centric Functional Modeling has been used to define a hypothetical model for the complete set of behaviors that the human consciousness system can possibly have, that is, a model of all possible awarenesses 
and awareness processes (processes by which human consciousness might transition from one awareness to another) that human consciousness can possibly experience. This hypothetical awareness space or space of awareness's (also called the "consciousness space") is similarly an open graph containing a network of all possible awarenesses in which each awareness is connected to other awarenesses by awareness processes.

Assume that awareness (experience) has some relationship with truth. For now we will assume that awareness of truth shares sufficient properties in common with truth that we can consider awareness and truth to be the same. Then assuming that this graph can contain all possible awarenesses and awareness processes, it is a complete representation of the human meaning of awareness or experience of any truth, that is, a complete semantic model of the experience of truth.

In general, it is hypothesized that Human-Centric Functional Modeling can always be used to define a model for our thoughts, as well as a model for the real-world systems that we can think about. In this paper we assume that this capacity for dual representation can be extended to define a semantic model that might be used to represent all possible information, and therefore all possible mathematical logic and all possible mathematical proofs by which that logic might be demonstrated to be valid, as well as to define a model for the system of all possible truths (such as mathematical relationships) that we might think about. Here we assume that information is some entity that is distinct from truth. As mentioned, we also assume that the hypothetical conceptual space (the functional state space of the cognitive system) used to represent this information is a semantic model with the capacity to represent all possible information, and we assume this awareness space (the functional state space of the consciousness) is a semantic model with the capacity to represent all possible awareness of truth, and therefore all possible truth in so far as truth and awareness are equivalent.

\section{Representing Computable and Uncomputable Information in Functional State Space}

In the conceptual space that represents the functional state space of the cognitive system, the term "computable information" describes any concept that can be reached through some logical reasoning process, and is represented in conceptual space by paths that can be constructed in terms of other paths. The term "uncomputable information" describes any concept that can only be reached through intuition, pattern recognition, or some other reasoning process that assumes the solution, and is represented in conceptual space by direct paths that cannot be constructed in terms of other paths. In cognitive psychology uncomputable reasoning (reasoning used to determine uncomputable information) is referred to as type 1 or intuitive reasoning, and computable reasoning (reasoning used to determine computable information) is referred to as type 2 or rational methodical reasoning [4]. One benefit of defining this conceptual space is that it is easy to see intuitively in the conceptual space model that if the graph is not continuous, then there will always be some separation between one concept and another, and there will not be any reasoning that the smallest reasoning paths can be broken down into. It would appear then that all computable reasoning is eventually based on uncomputable reasoning. While this remains to be proven, paradoxically at the same time it is important to recognize this because it clarifies the argument that no mathematical proof can exist without any assumptions.

\section{Representing Relative and Absolute Truth in Functional State Space}

Investigating the meaning of "truth", in functional state space truth is hypothesized to have two representations, one being relative truth, which is dependent on context, assumptions, or other factors, and absolute truth. Relative truth has been represented here as a signal. From the perspective of Human-Centric Functional Modeling, any given reasoning processes in conceptual space that is eventually based on uncomputable reasoning is eventually based on assumptions. Any reasoning 
process or any given process in any other functional state space, has been represented as a channel that conveys truth as a signal with some degree of error that is hypothesized to be given by information theory [9]. In other words, in conceptual space the relative truth of one claim can be defined in terms of the degree of self-consistency of the group of claims contained in the generalization of that claim, where self-consistency is a measure of the ability of that claim to transmit truth as a signal. As an example, to assess the truth of the claim, a "pig is smaller than a horse", we can generalize the concept "pig" to include all breeds of pigs, and we can generalize "horse" to include all kinds of horses. We can then generalize the reasoning "is smaller than" to include all the processes by which we determine any animal to be smaller than any other, and we can apply them to every combination of pig and horse in these two sets. This set of reasoning connecting all the concepts in these two sets of generalizations defines a channel through which information flows. If the truth of each specific reasoning process linking each specific breed of pig or horse is a signal, then a poor channel will permit the transmission of little signal and much noise. Whereas a good channel will permit the transmission of much signal little noise. This is depicted in figure 1.
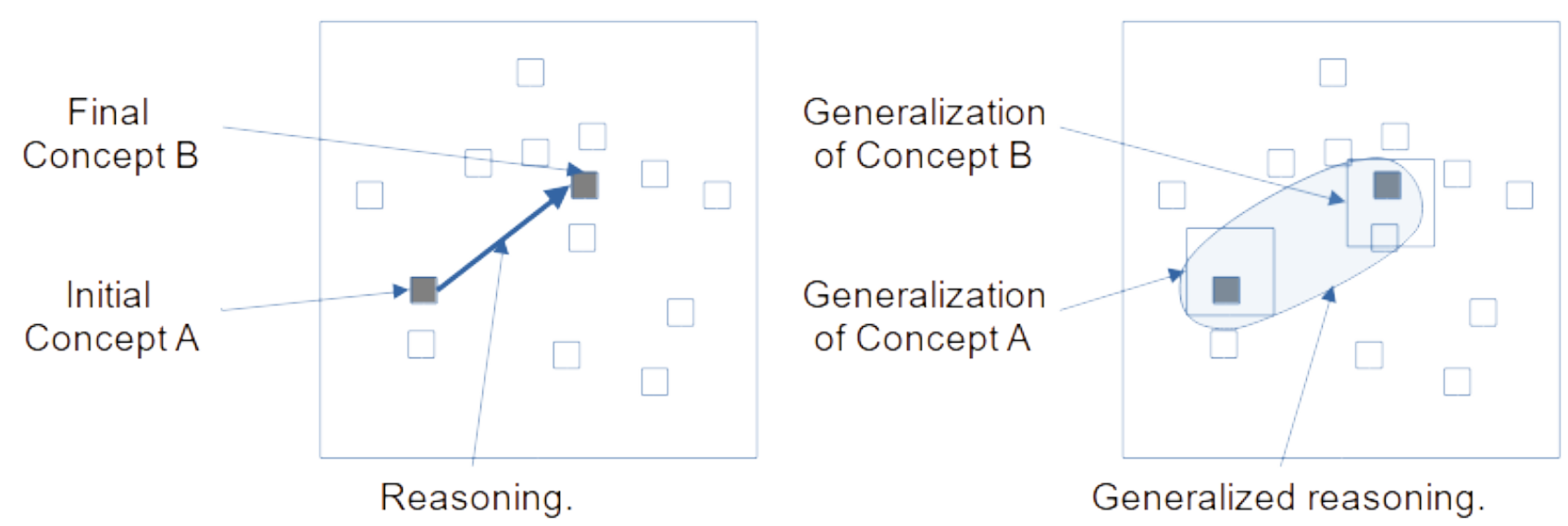

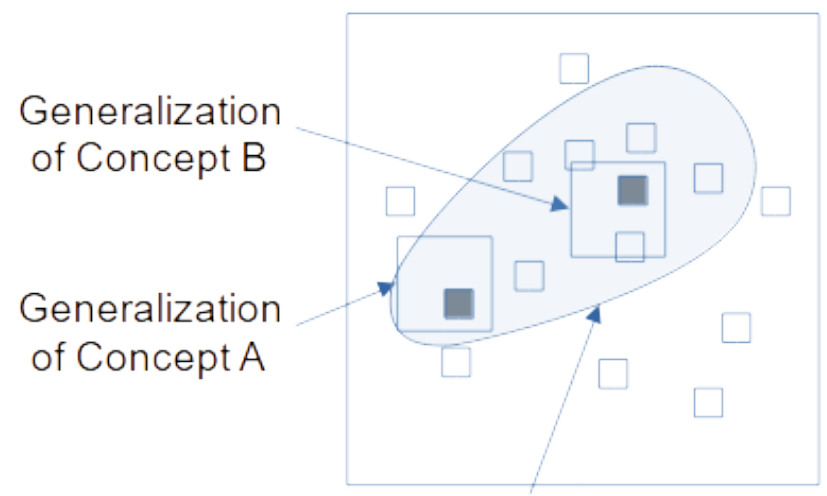

Generalized reasoning.

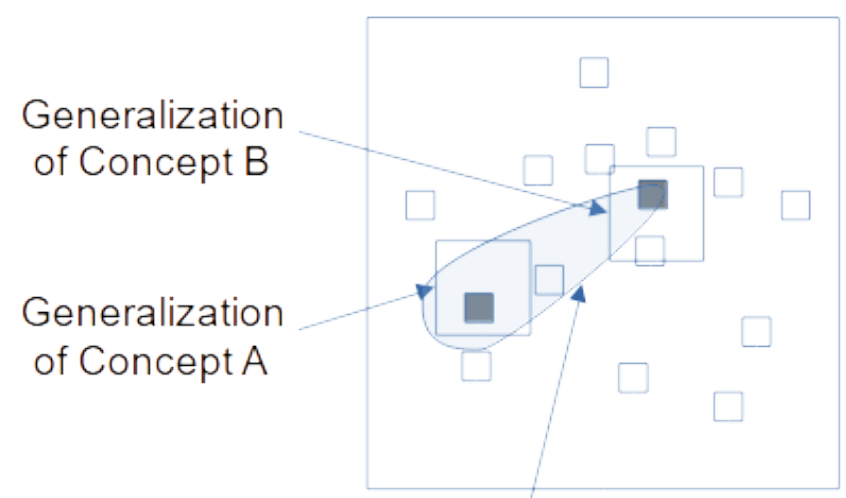

Generalized reasoning.

Figure 1: Truth in Functional State Space. Top left: reasoning representing a claim of unknown truth that connects some concept $A$ and some concept B. Top right: generalized reasoning representing a claim that is true in the sense that all reasoning processes that can be generalized as belonging to the same set are consistent in tracing a path between generalizations of the same concept $A$ and B. Bottom left: generalized reasoning representing a claim that is partially true in the sense that some reasoning processes that can be generalized as belonging to the same set are not consistent and trace a path from the generalization of concept $A$ but do not end up within the generalization of concept $B$. Bottom right: generalized reasoning representing a claim that is completely true but that is only part of the truth in 
the sense that all reasoning processes that can be generalized as belonging to the same set are consistent in tracing a path between generalizations of the same concept A and B, but some of the paths between those concepts are outside that generalized reasoning process.

Another representation of truth is absolute truth. Any continuous geometry defined in a functional state space is hypothesized here to represent an absolute truth in that for all statements about that geometry, because continuous lines are represented by real numbers, and because there is always a real number between any two real numbers, then associating the length of reasoning paths with real numbers, there is always some reasoning enclosed in that geometry that any given reasoning in that geometry can be constructed from. One need never fall back on assumptions. If any given path within that geometry is true, and if that geometry is continuous, the set of paths proving that truth must be contained within that functional state space, and therefore must be provable within that geometry. These statements regarding the definition of absolute truths in functional state space remain to be proved. However, once relative or absolute truths can be defined in any functional state space and identified with regions in the graph of functional state space or identified with continuous geometries, then relationships can be defined between those truths. One set of relationships between truths are (geometric) transforms in functional state space from one truth to another. Define a transform to be "true" if all entities that are "true" in the absolute or relative sense are also transformed to be true in the absolute or relative sense respectively. Then any reasoning that is "true" in terms of being a solution to any one problem might be generalized through such transforms to any other problem where it applies ("applies" in the sense that it is also true). An example is provided in figure 3.

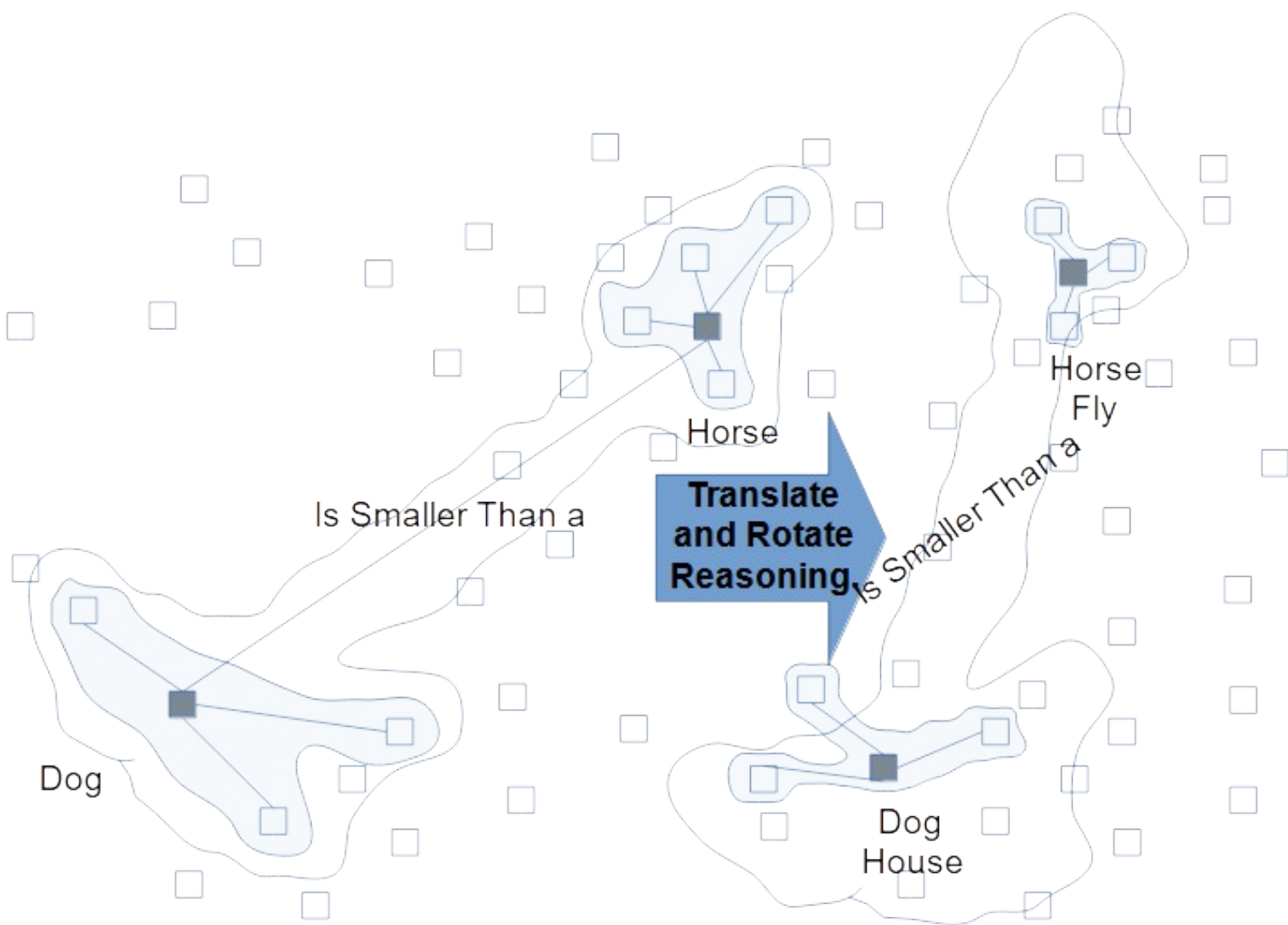

Figure 3: Some geometric transformations might be applied to the geometry of a claim in conceptual space (e.g. the geometry might be translated and rotated) in conceptual space and applied to a new set of concepts in that new region of conceptual space. Some geometries when transformed to a different 
region of conceptual space are no longer "true" as in the above case in which the geometry of the claim "a dog is smaller than a horse" is transformed to begin at the concept "dog house" and to end at the concept "horse fly". In this second case the claim does not encompass the required initial and target concepts and therefore is not "true".

Geometries might be defined independently of concepts themselves in conceptual space to represent absolute truths. However, the equivalence of open functional state spaces suggests that the uncertainty principle which governs physical space also has some equivalent in other open functional state spaces such as those hypothesized to represent cognition or consciousness. If the resolution of functional state spaces is given by the resolution of the functional states in those spaces, and if the resolution has fundamental limits such as due to uncertainty, then every known functional state space is limited in the resolution at which it is able to represent the truth of a geometry. Consider a diamond shaped region in some functional state space such as the functional state space representing matter, or the functional state space representing cognition (figure 4).

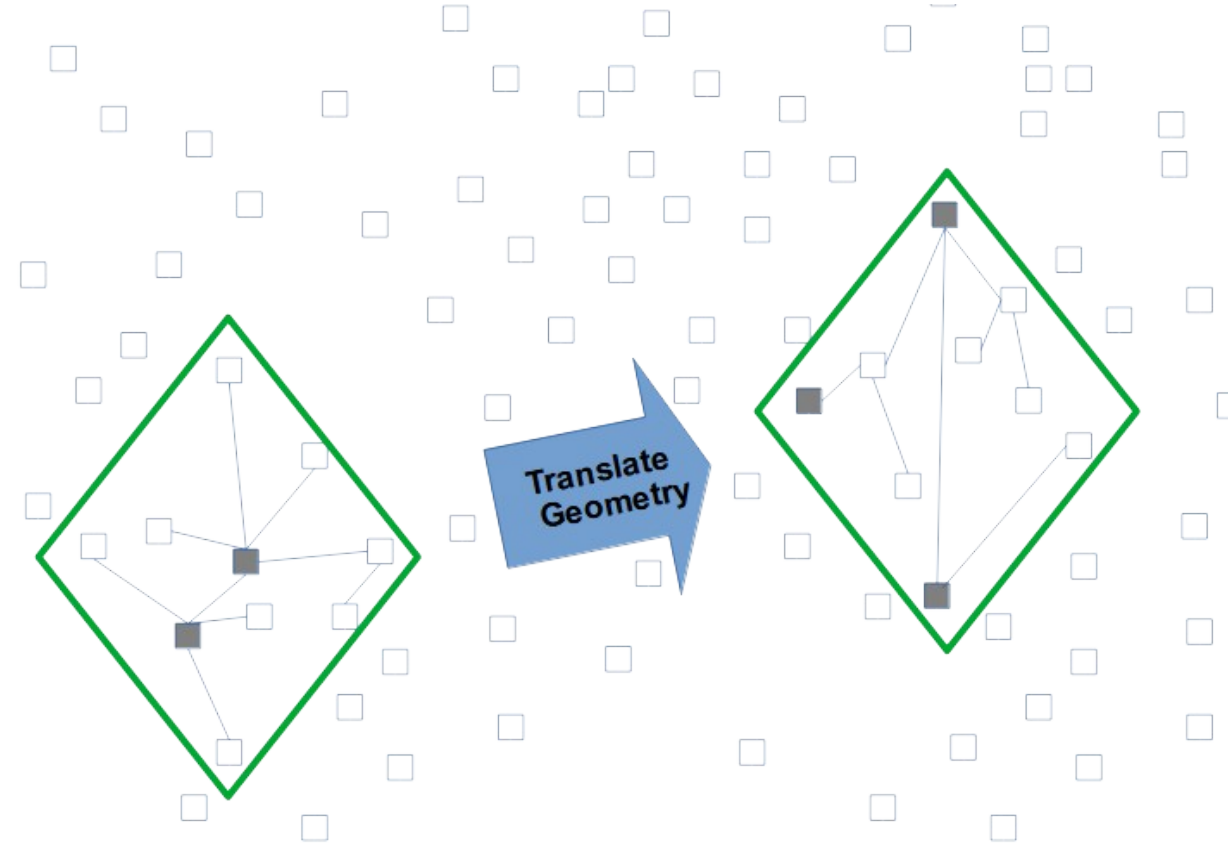

Figure 4: The truth in a geometry (green diamond shape) might be transformed to a different position in functional state space. This truth is "absolute" in being independent of the concepts and reasoning in any given region of conceptual space.

In both cases, the absolute truth described by such a geometry is a limit of any relative truth described by the graph because a smooth geometry can only be achieved with infinite resolution in that functional state space. Since resolution is limited in the real world by the Planck distance, it is a limit that potentially cannot be reached in any real system.

\section{Mathematical Relationships and Relative and Absolute Truth}

If relative truth is represented in conceptual space by some region in the graph of conceptual space, and if absolute truth is represented by some geometry in conceptual space, how are mathematical logic, and experience of mathematical truth that might be expressed by mathematical relations all related? First we must qualify what constitutes mathematical logic and what constitutes mathematical truth. We propose, that to be well defined, mathematical logic or mathematical truth must have a well defined sequence as in figure $\mathrm{x} 1$. 

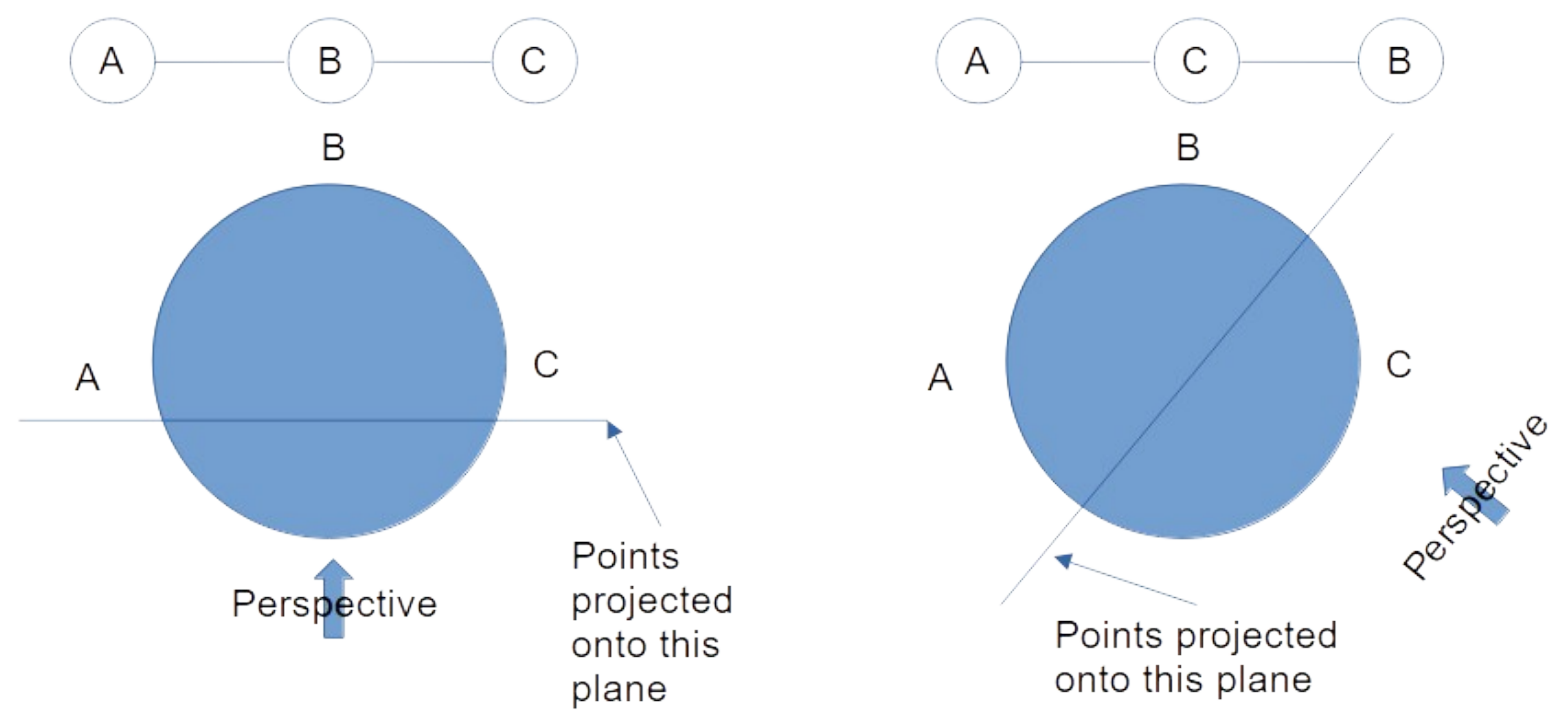

Figure x1: If mathematical logic or any experience of mathematical truth is represented by some region in the graph of some functional state space (blue circle), then depending on the perspective from which that graph is viewed the sequence of logic or experience might change.

Mathematical logic and mathematical truth are also defined by the structure and position of the corresponding paths in functional state space as in figure $\mathrm{x} 2$.

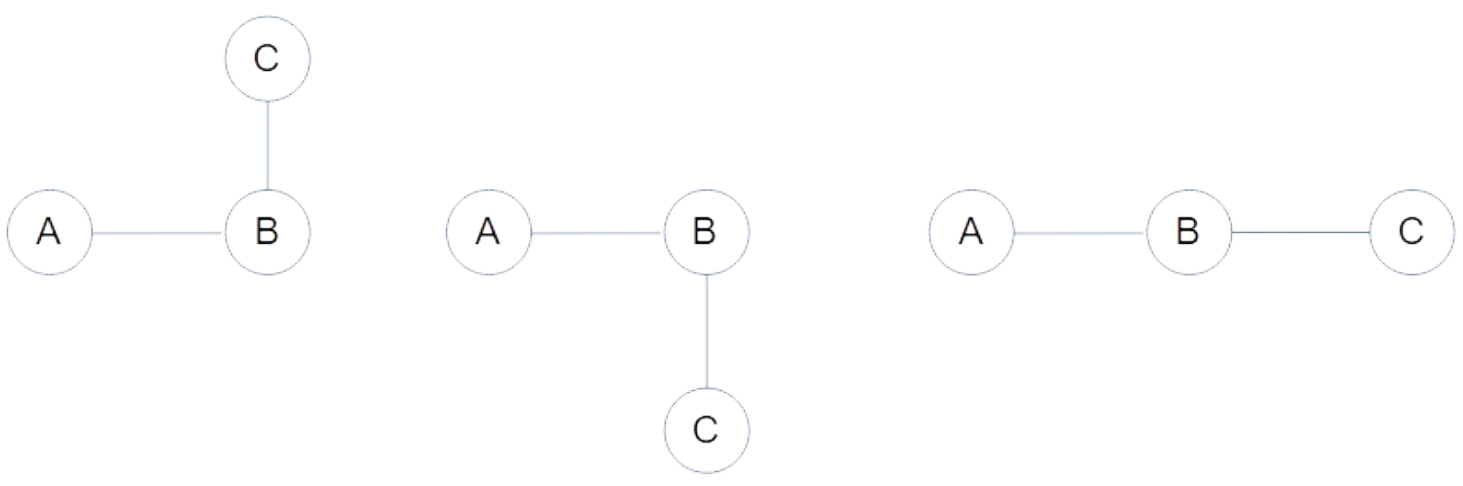

Figure x2: If information has position in functional state space, then reasoning has distance in functional state space. If so then mathematical logic can be qualitatively understood as being in line with, opposite from, or a sharp departure from. Left: $C$ is a departure from the line of reasoning $A-B$. Middle: $C$ is a departure in the opposite direction. Right: $C$ is an extension in reasoning that is line with $A-B$. Experience of mathematical truth can be qualitatively understood in the same way.

To be well-defined this mathematical logic or mathematical truth must have a well-defined sequence as in figure $\mathrm{x} 3$. 

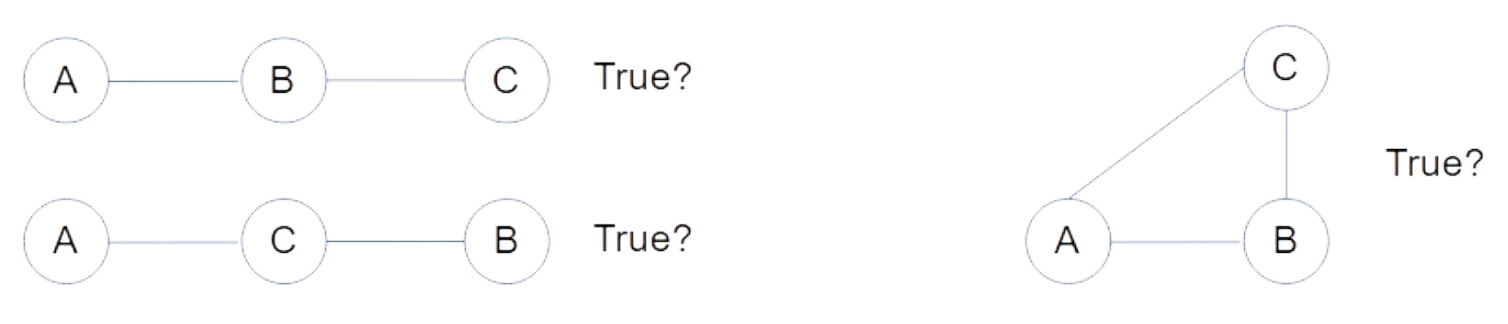

Figure x3: If the sequence of mathematical logic or the sequence of experience of mathematical truth are indeterminate, this indicates that some higher dimensionality exists. In this example, indeterminacy in the sequence of mathematical logic in one dimension is resolved by representing the logical relationships in two dimensions.

Human-Centric Functional Modeling is in essence a mathematical representation of our perception (sensory perception, emotional perception, cognitive perception, and conscious perceptions) as fields modeled in terms of network graphs. Rather than being focused on external measurement, this mathematical representation of perception is focused on the first-person observation characteristic of Eastern existential philosophies. Because all humans share the same perceptual spaces, this representation in terms of first person observation facilitates convergence science. As an example of one way in which it does so, in mathematics one might try to understand the set theory from the perspective of graph theory. Wherever graph theory does not translate into set theory, graph theory cannot be understood using this method. However, if one understands graph theory in terms of paths through conceptual space, conceptual space contains all concepts and reasoning and therefore can always be used to acquire this understanding. As an example of another way in which functional state spaces facilitate convergence science, all truths can potentially be idealized as geometries in the sense that functional states and the processes defining relationships between functional states can be represented in the limit of continuous functional states spaces by continuous geometries. Geometric transforms (translation, reflection, etc.) of these geometries can then enable truths from one region in conceptual space or in any other functional state spaces, to be used to discover truths in other regions or even in other functional state spaces.

One of the main goals of the functional state space approach in fact is that patterns used to radically increase problem-solving ability in one functional state space can potentially be reused to radically increase problem-solving ability in other functional state spaces. As an example, decoupling all processes in functional state space representing all possible transitions into a library used to significantly increase problem-solving ability is a pattern used in conceptual space to decouple all AI processes representing all possible reasoning in conceptual space into a library that an Artificial General Intelligence (AGI) might use to radically increase its problem-solving ability, enabling humans to radically increase their capacity to solve the problem of AGI [8]. The same radical increase in capacity to understand and discover mathematical relationships is predicted to be possible if functional state spaces can be applied to mathematical logic and to mathematical truth.

The relationship between Human-Centric Functional Modeling and the amazing proclivity of some Eastern mathematicians to discover new mathematical relationships without formal training, such as displayed by the mathematician Ramanujan [8], might be an interesting line of inquiry in terms of reviewing his work to detect any signature of this functional state space based reasoning style, which would appear to be a natural progression from Eastern existential philosophies. Srinivasa Ramanujan 
(22 December 1887 - 26 April 1920) [8], was a gifted layman who was able to derive a number of complex mathematical theorems largely without a formal education in mathematics. In functional state space at least one aspect of creativity is potentially an algorithm in which one generalizes complex relationships and reuses them in other domains where they apply. This paper speculates that Ramanujan and other such individuals might have developed a functional model of mathematics that enabled them to in essence leverage an algorithm for creativity, that is, an algorithm for generalizing complex relationships and reusing them in other domains where they applied. What amounts to functional modeling has been proposed to be a fundamental part of the Vedic culture of which Ramanujan was a devoted follower. Because functional modeling describes systems in terms of functions and the dynamics between those functions, complex systems can be understood even where the mechanisms by which those functions are implemented are unknown.

\section{Implications}

There are a number of implications to the theory that for any logic defining any mathematical relationship, in the limit of infinite cognitive ability that logic is a geometry in some functional state space. And there are a number of implications to the theory that for any truth represented by any mathematical relationship, in the limit of infinite conscious awareness that truth is a geometry in some functional state space.

Consider the case of a logical argument that is an ordered sequence of claims that can be idealized as paths on some geometry, where that geometry is represented by some function. Consider also a mathematical relationship to share some property in common with the as yet undefined entity "truth" such that we can treat the mathematical relationship as a proxy for truth. Consider the case of a mathematical relationships that can be projected onto any $\mathrm{N}$ dimensional space, whether that space is of zero curvature (Euclidean space), positive curvature (hyperbolic space), or negative curvature (elliptic space).

As in figure $\mathrm{x} 3$, if a path projected onto some space represents an ordered sequence of claims, or if a path projected onto some space represents a relationship, and if the sequence of points separating the segments along that path and therefore the segments along that path in a geometry themselves are indeterminate (change depending on the perspective taken for the projection), then the relationship may exist in a space of higher dimensionality than that upon which it has been projected.

In the limit of infinite reasoning ability, mathematical reasoning such as proofs can potentially be represented as a continuous geometry in a functional state space, specifically, in the conceptual space representing reasoning. In the limit of infinite awareness, mathematical truth can potentially be represented as a continuous geometry in a functional state space, specifically, in the awareness space representing awarenesses. The concept of logical proofs being a geometry in conceptual space is important because it allows one to determine the logical proofs that can't be defined or discovered because the geometry of those proofs is outside the ability of our cognition to detect. This might happen when the geometry is past the resolution we can detect or past the size we can detect. The concept of truth being a geometry in awareness space is important because it allows one to determine the truths that can't be defined or discovered because the geometry of those truths is outside the ability of our consciousness to detect.

The concept of mathematical logic and mathematical truth being geometries in functional state spaces is important because once a representation of these functional state spaces can be defined, and once sufficient mathematical logic and mathematical truth to be able to map to any geometry can be modeled, this potentially allows one to define new mathematical problems and to discover new 
mathematical solutions using geometric transformations of those geometries. The projection or mapping of one geometry in one space to another geometry in another space involves the transformation of one geometry to another, as well as the mapping of one space to another. It may be that the functional state space representing the cognition or the functional state space representing the awareness might have higher dimensions. If so, the mapping to those higher dimensions and the transformation between geometries might be necessary to represent all capabilities of human consciousness and cognition.

Branching in functional state space represents the fact that response to information or truth is nondeterministic. Following the hypothesis that functional state spaces can be used to represent all systems, including the physical universe or any object in it, a functional state space has already been conceptualize that represents any possible information about the state of any object in the universe, and another functional state space has already been conceptualize to represent any possible truth regarding the awareness of the state of any object in the universe [7]. It is clear that all possible statements of information or truth should include specification of the time. Assuming both time and physical distance has some representation in functional state space, and assuming that any representation of a property one functional state space is potentially the same in every other functional state space, then is it is also clear that the ambiguity of the concept of simultaneity and the ambiguity of relative distance in physical space suggests that the duration and order of logic in conceptual space are also inherently ambiguous, and in addition suggests that the duration and order of awarenesses (the duration and order in which truth is experienced) are inherently ambiguous as well. In other words, there is likely a kind of relativity in functional state space. Assume logic and truth have some sequence, and that if this sequence changes the logic or truth themselves change. This paper has hypothesized that if logic or truth exist in higher dimensions than an observer percieves (that is, if they exist in some hyperspace), then if there is any change in one's perspective in this hyperspace, logic or experience of truth themselves might change since their order could be different based on one's point of view. The theory of relativity also functions to show how the order of logic or experience might change. This raises the question of whether there is any relationship between higher dimensions in functional state spaces and relativity.

Furthermore, in physics there are three ways of thinking. Newtonian in which the order of events is absolute regardless of perspective, relativistic in which based on one's point of view the order of events changes, and quantum mechanical in which ascertaining what is the precondition and what is the result is indeterminite because states are probablistic. If one statement of logic or one experience of truth can't be said to come before another then the sequence of logic or experience of truth does not exist. This raises the question of whether there is any relationship between higher dimensions in functional state spaces and quantum mechanics as well as a relationship with relativity. Such a relationship is in fact suggested by the "structure" within uncertainty that is assumed in a representation of the physical universe from the Human-Centric Functional Modeling perspective [7].

\section{Questions for Future Study}

In physics it has been hypothesized that in representing physical systems an awareness can be true in that one particle can potentially receive that awareness. In mathematics it has been hypothesized here that there is some limit in which every awareness is a geometry. If so, then what geometries of awareness are potentially true? What mathematical relationships define the boundaries of those awareness's? If a geometry can be defined in any $\mathrm{N}$ dimensional space of positive curvature, of zero curvature, or of negative curvature, then if truth is a geometry how can all these truths in all these spaces be represented? 
Regarding information, a concept cannot be true or false. It either exists or does not exist. However, the semantic representation of any concept can be true in the sense that it can be a valid representation in the semantic modeling framework being used. Regarding mathematical logic or any other reasoning on the other hand, that reasoning might exist or might not exist, but can also be true or false. Each concept has a location in conceptual space. Each set of reasoning has a shape in conceptual space.

Awarenesses (experiences) are different from concepts in that while each awareness or experience might or might not exist, if an awareness exists then it shares some property of truth and might be considered to be true. Where every element of information differs in being defined through different reasoning, every element of awareness shares in common the fact that it is true. This suggests that concepts or ideas function very differently from awareness or experience. If mathematical reasoning is a knife that slices information into ever finer distinctions, then does awareness of mathematical truth provide a path towards some deeper unity in which experience of any one mathematical truth can be directed towards experience of any other?

\section{Conclusions}

Both relative and absolute truth as defined in this paper are hypothesized to exist in both conceptual space and awareness space. One difference is that truth in conceptual space pertains to truth of reasoning, and truth in awareness space pertains to truth of experience processes. If experience itself has properties shared by truth, aside from the truth in the experience processes that connect such experiences, and if as a consequence experience or awareness can be equated with truth under at least some conditions, then another difference is that it is hypothesized that concepts cannot be true or false. The awareness space would seem to be far more important to mathematics because while it is hypothesized that all reasoning in conceptual space must at root rely on assumptions, all experience potentially shares some common properties with truth. By recognizing the mathematical truths associated with any given geometry in awareness space, and by understanding how geometric transforms might be applied to move those mathematical truths to different regions of awareness space, it may be possible to achieve a radical increase in ability to discover new mathematical truths from existing ones.

The semantic model for information provided by Human-Centric Functional Modeling might be incorrect, but if the human meaning of information exists then, SOME semantic model for information exists that is valid. If ANY semantic model of information is valid, then all representations of mathematical logic are valid representations in that model even though they might not represent valid logic. Similarly, the semantic model for truth provided by Human-Centric Functional Modeling might be incorrect, but if the human meaning of truth exists then, SOME semantic model for truth exists that is valid. If ANY semantic model of truth is valid, then all representations of mathematical truth are valid representations in that model even though they might not represent valid truth within our awareness. Assuming that any semantic model of information defined using network theory must define distances and relative position, then any real-world representation of information has both a finite resolution and a structure in this model. Assuming that a problem represents a gap between two points in the conceptual space representing any given information, and that a solution is represented in conceptual space by the reasoning or understanding processes which provide that path, then this resolution represents the finite ability of the cognitive system to define problems or to discover and differentiate solutions. However, in the limit of infinite resolution, discrete points in conceptual space, become lines, and networks become continuous geometries. Therefore if logic can be represented by a graph defined through network theory, in this limit of infinite resolution logic is hypothesized to be replaced by a geometry. Similarly, if awareness of truth can be represented by a graph defined through 
network theory, in this limit of infinite resolution truth itself is hypothesized to be replaced by a geometry.

In summary, with regards to logic, one set of logic represented in terms of a set of mathematical claims might not be consistent or even expressible in terms of another. Because a functional approach only represents the outcomes that a model functions to achieve, rather than attempting to define or discern a model in terms of other models, then all possible mathematical logic can potentially be represented. Both the completeness of capacity to represent all mathematical logic and the claim that in the limit of infinite reasoning ability mathematical logic transitions from a graph to a geometry remain to be proven.

With regards to truth, truth might act fundamentally differently from information in ways that remain to be defined. However, again, because a functional approach only represents the outcomes that a model functions to achieve, rather than attempting to define or discern a model in terms of other models, then all possible mathematical truth can potentially be represented without having to define truth. Again, both the completeness of capacity to represent all mathematical truth and the claim that in the limit of infinite awareness mathematical truth transitions from a graph to a geometry remain to be proven. Because the solutions to wicked problems of optimization such as potentially poverty or the solutions to wicked problems of dynamical stability such as potentially sustainability and sustainable economic development are hypothesized to be patterns in functional state space [6], and because professionals in those sustainable development goals might in general lack the mathematical language to address these fundamental questions, then motivating mathematicians to study these issues is of existential importance.

\section{Acknowledgments}

Many thanks to Julian Talev for endless discussions and for many valuable contributions on this topic.

\section{References}

[1] Biggs, N.; Lloyd, E.; Wilson, R. (1986), Graph Theory, 1736-1936, Oxford University Press

[2] Newman, Mark (2010). Networks: An Introduction (PDF). Oxford University Press.

[3] Williams, Andy E. "Human-Centric Functional Modeling and the Unification of Systems Thinking Approaches: A Short Communication." Journal of Systems Thinking (2021): 5-5.

[4] Kahnemen, Daniel. "Thinking fast and slow." New York: Farrar, Straus and Giroux (2011).

[5] Kanigel, Robert (1991). The Man Who Knew Infinity: a Life of the Genius Ramanujan. New York: Charles Scribner's Sons. ISBN 978-0-684-19259-8.

[6] Williams, A.E. Are wicked problems a lack of general collective intelligence?. AI \& Soc (2021). https://doi.org/10.1007/s00146-021-01297-8

[7] Williams, A. E. (n.d.). The Importance of Representing the Physical Universe or Virtual Universes in Functional State Space to the Progress of Physics. Retrieved from osf.io/preprints/africarxiv/54qrz [8] Williams, A. E. (2020, April 27). Defining Functional Models of Artificial Intelligence Solutions to Create a Library that an Artificial General Intelligence can use to Increase General Problem Solving Ability. https://doi.org/10.31730/osf.io/hpzb7

[9] Shannon, C.E. (1948), "A Mathematical Theory of Communication", Bell System Technical Journal, 27, pp. 379-423 \& 623-656, July \& October, 1948 